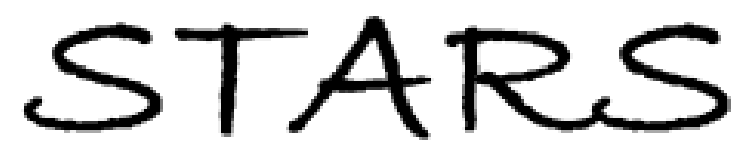

University of Central Florida

STARS

$1-1-1996$

\title{
Angular Distribution Of Decay Electrons From The 6P Resonance Excitations In Xenon
}

\author{
C. D. Caldwell \\ University of Central Florida \\ S. Hallman \\ University of Central Florida
}

Find similar works at: https://stars.library.ucf.edu/facultybib1990

University of Central Florida Libraries http://library.ucf.edu

This Article is brought to you for free and open access by the Faculty Bibliography at STARS. It has been accepted for inclusion in Faculty Bibliography 1990s by an authorized administrator of STARS. For more information, please contact STARS@ucf.edu.

\section{Recommended Citation}

Caldwell, C. D. and Hallman, S., "Angular Distribution Of Decay Electrons From The 6P Resonance Excitations In Xenon" (1996). Faculty Bibliography 1990s. 1583.

https://stars.library.ucf.edu/facultybib1990/1583

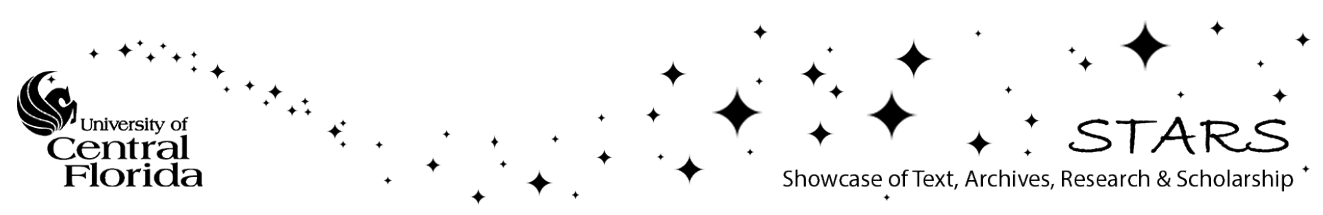




\title{
Angular distribution of decay electrons from the $6 p$ resonance excitations in xenon
}

\author{
C. D. Caldwell and S. Hallman \\ Department of Physics, University of Central Florida, Orlando, Florida 32816-2385
}

(Received 14 December 1995)

\begin{abstract}
Angular distributions for the electrons produced by the decay of the $4 d \rightarrow 6 p$ excitations in xenon have been measured using an undulator radiation source. The resolution employed in the experiment has allowed us to derive separate $\beta$ values for features which had not been isolated previously, as well as to determine $\beta$ values for previously unreported lines. Comparison is made between existing experimental and theoretical results.
\end{abstract}

PACS number(s): $32.80 . \mathrm{Fb}, 32.80 . \mathrm{Hd}$

The $4 d \rightarrow 6 p$ excitations in xenon represent one of the best-known examples of resonant Auger decay. Although the excitation is to an unfilled principal shell, the decay spectrum is quite complex due to strong spin-orbit coupling in the xenon atom. This complexity demands that a sufficiently high resolution be employed in order to resolve the contributions from each of the spin-orbit components. To date no truly high-resolution data have been reported for the angular distribution parameter $\beta$ associated with the decay of these excitations. The highest resolution measurements thus far are the cross section results of Aksela et al. [1].

In this paper we report on an analysis of the angular distribution of the resonant Auger electrons resulting from the decay of both the $4 d_{5 / 2}^{-1} 6 p$ and $4 d_{3 / 2}^{-1} 6 p$ excitations. The data for this analysis are the result of experiments conducted at the University of Wisconsin Synchrotron Radiation Center (SRC) using an undulator-monochromator combination
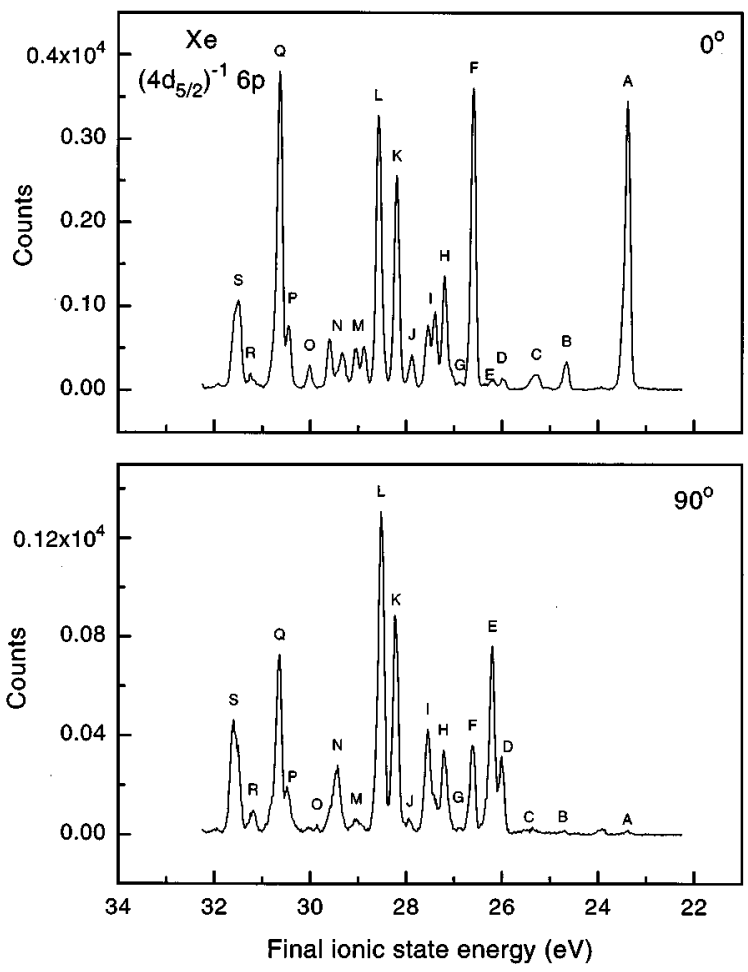

FIG. 1. Intensities of the electrons resulting from the decay of the $4 d_{5 / 2}^{-1} 6 p$ excitation in xenon as measured at $0^{\circ}$ and $90^{\circ}$. Labels correspond to the groups of lines given in Table I. which provided radiation with a bandwidth of $60 \mathrm{meV}$ at the energies of interest for the xenon measurements. Details of the operation of this source and the experimental arrangement have been discussed extensively in an earlier work [2]. Briefly, the electrons are analyzed in a set of three spherical sector analyzers mounted at right angles with respect to each other on a rotatable platform perpendicular to the direction of the incoming photon beam. The angular distribution parameter $\beta$ is obtained from the ratio of photoelectron intensities at $0^{\circ}$ and $90^{\circ}$ with respect to the electric field vector, $R=I\left(0^{\circ}\right) / I\left(90^{\circ}\right)$, through the relation

$$
\beta=\frac{4(R-1)}{3 p(R+1)-(R-1)},
$$

where $p$ is the polarization of the ionizing radiation; in this case $p=0.99$. A measurement at the pseudomagic angle of

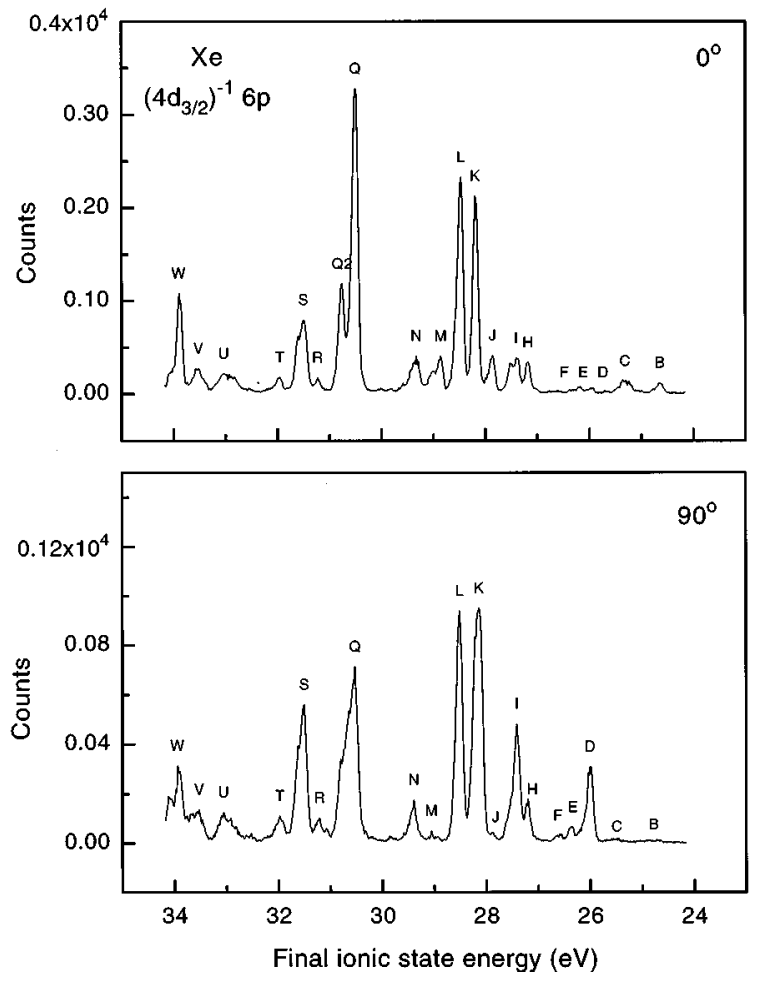

FIG. 2. Intensities of the electrons resulting from the decay of the $4 d_{3 / 2}^{-1} 6 p$ excitation in xenon as measured at $0^{\circ}$ and $90^{\circ}$. Labels correspond to the groups of lines given in Table II. 
TABLE I. Experimental and theoretical $\beta$ values for $4 d_{5 / 2}^{-1} 6 p$ excitations in xenon. Lines are a combination of transitions identified by Aksela et al. (Ref. [1]), given in column 2. Designations used by Carlson et al. (Ref. [4]) and Chen (Ref. [7]) are given in column 3.

\begin{tabular}{|c|c|c|c|c|c|c|c|c|}
\hline \multirow[b]{2}{*}{ Line } & \multicolumn{2}{|c|}{ Designation } & \multirow{2}{*}{$\frac{\text { This work }}{\beta}$} & \multicolumn{3}{|c|}{ Experimental } & \multicolumn{2}{|c|}{ Theoretical } \\
\hline & Aksela & Carlson & & $\beta^{\mathrm{a}}$ & $\beta^{\mathrm{b}}$ & $\beta^{\mathrm{c}}$ & $\beta^{\mathrm{d}}$ & $\beta^{\mathrm{e}}$ \\
\hline$A$ & 1 & & $1.96(1)$ & & & & & \\
\hline$B$ & 7,8 & & $1.69(8)$ & & & & & \\
\hline C & $12-14$ & & $1.5(1)$ & & & & & \\
\hline$D$ & 19,20 & $1 \mathrm{a}$ & $-0.59(6)$ & -0.88 & -0.67 & -0.60 & -0.976 & -0.770 \\
\hline E & $22-24$ & $1 b$ & $-0.86(3)$ & -0.93 & -0.93 & -0.90 & -0.726 & -0.870 \\
\hline$F$ & 26 & $1 \mathrm{c}$ & $1.38(6)$ & 0.82 & 1.35 & 1.31 & 0.972 & 1.018 \\
\hline$G$ & 27 & & $1.1(2)$ & & & & & \\
\hline$H$ & 30,31 & $2 a$ & $0.84(9)$ & 0.26 & 0.89 & 0.58 & 0.868 & 0.703 \\
\hline$I 1$ & 32,33 & & $1.17(9)$ & & & & & \\
\hline$I 2$ & $34-36$ & & $0.2(1)$ & & & & & \\
\hline$I$ & $32-36$ & $2 b$ & $0.59(9)$ & 0.16 & 0.45 & 0.54 & 0.394 & 0.348 \\
\hline$J$ & 37,38 & & $1.4(1)$ & & & & & \\
\hline K & $39-42$ & $3 a$ & $0.56(8)$ & -0.02 & 0.55 & 0.23 & -0.048 & 0.039 \\
\hline$L 1$ & 43,44 & & $-0.79(3)$ & & & & & \\
\hline$L 2$ & 46 & & $1.74(3)$ & & & & & \\
\hline$L$ & $43-46$ & $3 b$ & $0.45(8)$ & -0.09 & 0.46 & 0.33 & 0.007 & -0.0007 \\
\hline$M 1$ & 48,49 & & $1.66(7)$ & & & & & \\
\hline$M 2$ & 50 & & $1.4(1)$ & & & & & \\
\hline$M$ & $48-50$ & & $1.52(8)$ & & & & & \\
\hline$N 1$ & $51-55$ & & $0.3(1)$ & & & & & \\
\hline$N 2$ & 56 & & $1.3(1)$ & & & & & \\
\hline$N$ & $51-56$ & & $0.6(1)$ & & & & & \\
\hline$O$ & 59 & & $1.5(1)$ & & & & & \\
\hline$P$ & 63,64 & & $1.0(1)$ & & & & & \\
\hline$Q 1$ & 67,68 & & $1.04(8)$ & & & & & \\
\hline$Q 2$ & 72 & & $0.7(2)$ & & & & & \\
\hline$Q$ & $67-72$ & 5 & $0.95(8)$ & 0.51 & 1.26 & 0.83 & 0.753 & 0.847 \\
\hline$R$ & $74-79$ & & $0.3(1)$ & & & & & \\
\hline$S 1$ & $80-82$ & & $0.8(1)$ & & & & & \\
\hline$S 2$ & $83-85$ & & $0.2(1)$ & & & & & \\
\hline$S$ & $80-85$ & 6 & $0.47(9)$ & -0.03 & & 0.12 & -0.01 & 0.017 \\
\hline
\end{tabular}

${ }^{\mathrm{a}}$ Carlson et al. (Ref. [4]).

${ }^{\mathrm{b}}$ Hergenhahn et al. (Ref. [5]).

${ }^{\mathrm{c}}$ Kämmerling et al. (Ref. [8]).

${ }^{\mathrm{d}}$ Hergenhahn et al. (Ref. [6]).

${ }^{\mathrm{e} C h e n ~(R e f . ~[7]) . ~}$

$\theta_{m}=(1 / 2) \cos ^{-1}(-1 / 3 p)$, gives the partial cross sections. Energy resolution of the analyzers was enhanced by retarding the electrons to provide an overall experimental resolution of $147 \mathrm{meV}$, as measured by the width of the $5 s$ photoline. While this was adequate to resolve the major multiplet components, it was not sufficient to resolve all the spin-orbit components, which have a typical splitting of 20-30 meV. We employed two independent analyzers, which were rotated in tandem between the two angles, thereby allowing two independent measurements of the $\beta$ parameter.

In a recent measurement using a monochromator bandpass of $8 \mathrm{meV}$ and an electron spectrometer resolution of 40 meV, Aksela et al. [1] remeasured the decay spectra of the $4 d \rightarrow 6 p$ excitations at the magic angle. Using a combination of a fitting procedure and theoretical calculations, they assigned energies and relative intensities to all the spin-orbit components which they identified. Using their energies as a starting point, we fit each of our spectra, at $0^{\circ}$ and $90^{\circ}$, to a group of overlapping Pearson-7 functions [3] to determine the relative intensity of each line at each individual angle. Each spectrum was fit individually, and the goodness of the fits was determined by the $\chi$-squared value as well as the comparison between the two independent sets of data. Intensities for the various features are given by the area under the respective contributions which were summed to give the final fit, normalized to the beam current and the xenon pressure.

Figures 1 and 2 give the spectra for each of the excitations as recorded at the two angles. Each of the prominent features is labeled with a letter which corresponds to a group of spinorbit components making up a fit. In Tables I and II are given the results for the $\beta$ values which we determine for 
TABLE II. Experimental and theoretical $\beta$ values for $4 d_{3 / 2}^{-1} 6 p$ excitations in xenon. Lines are a combination of transitions identified by Aksela et al. (Ref. [1]). The other $\beta$ results are those of Carlson et al. (Ref. [4]).

\begin{tabular}{lccccccc}
\hline \hline & Designation & This work & Carlson & & Designation & This work & Carlson \\
\hline Line & Aksela & $\beta$ & $\beta^{\text {a }}$ & Line & Aksela & $\beta$ & $\beta^{\text {a }}$ \\
$B$ & 7,8 & $1.5(1)$ & & $M$ & $48-50$ & $1.75(5)$ & \\
$C$ & $12-14$ & $2.00(1)$ & & $N$ & $51-56$ & $0.6(1)$ & \\
$D$ & 19,20 & $-0.71(5)$ & -0.67 & $Q 1$ & $65-68$ & $0.7(1)$ & \\
$E$ & $22-25$ & $-0.03(1)$ & & $Q 2$ & $71-73$ & $0.6(1)$ & \\
$F$ & 26 & $-0.4(1)$ & -0.38 & $Q$ & $65-73$ & $0.62(9)$ & 0.89 \\
$H$ & 30,31 & $0.4(1)$ & 0.14 & $R$ & 76,77 & $0.4(2)$ & \\
$I 1$ & 32,33 & $-0.24(9)$ & & $S 1$ & $80-82$ & $0.1(1)$ & \\
$I 2$ & $34-36$ & $0.2(2)$ & & $S 2$ & $83-85$ & $0.2(1)$ & \\
$I$ & $32-36$ & $-0.13(9)$ & & $S$ & $80-85$ & $0.14(9)$ & -0.29 \\
$J$ & 37,38 & $1.53(9)$ & & $T$ & $86-91$ & $0.1(2)$ & \\
$K$ & $39-42$ & $0.0(2)$ & & $U$ & $94-100$ & $0.4(1)$ & \\
$L 1$ & 43,44 & $0.52(9)$ & & $V$ & $102-107$ & $0.3(1)$ & \\
$L 2$ & 46,47 & $-0.4(5)$ & & $W$ & $108-114$ & $0.5(1)$ & \\
$L$ & $43-47$ & $0.43(9)$ & 0.32 & & & & \\
\hline
\end{tabular}

${ }^{\mathrm{a}}$ Values in this column are for combinations of the line shown plus adjacent lines.

each of the groups of lines which we are able to isolate. In the second column of each table are given the numbers which Aksela et al. [1] have assigned to the individual lines having the energies which we calculate as making up the group. Because of the large number of spin-orbit components corresponding to most line numbers, we omit the detailed assignments and refer the reader to the Aksela work for details. The angular distribution parameter is calculated for each group by a direct division according to Eq. (1). In some instances in order to achieve a better comparison with earlier measurements and calculations, we have given both the $\beta$ values we determine for each distinguishable group and the average $\beta$ obtained when the two groups are superimposed.

The influence of resolution on these measurements is well illustrated by the behavior of feature $L$, which is expanded in Fig. 3 to show the individual contributions to the fit. Although the $K, L$ pair appears quite similar as recorded at the two different angles, closer examination reveals that there are actually several distinct features contained in $L$. The difference is highlighted by the relative contributions of lines $43-47$ [1] to the fit. At $0^{\circ}$ line 45 is a major contributor, whereas inclusion of line 44 makes no significant contribution. At $90^{\circ}$ the opposite is true. The contribution of line 46 is quite large at $0^{\circ}$ and relatively minor at $90^{\circ}$. Lines 43 and 44 are identified by Aksela et al. as $\left({ }^{1} D\right) 6 p^{2} D_{3 / 2,5 / 2}$, while line 46 is the $\left({ }^{1} D\right) 6 p^{2} P_{1 / 2}$ component. Lines 45 and 47 are the $\left({ }^{3} P\right) 7 s^{4} P_{5 / 2}$ and ${ }^{2} P_{3 / 2}$ components, respectively. These principal contributions can be separated into two larger features, one, $L 2$, with a large positive $\beta$ value and the second, $L 1$, with a large negative $\beta$ value. The final $\beta$ value in every case still remains an average, but the appearance of the two very different $\beta$ values for the two groupings illustrates the sensitivity of the final $\beta$ value to the relative intensities of the spin-orbit components making up the feature.

For the case of the $4 d_{5 / 2}^{-1} 6 p$ excitation, several sets of earlier data exist, both experimental and theoretical, for the $\beta$ values for the lines identified in the third column using the line numbering chosen by Carlson et al. [4]. These are intercompared in Table I. In general, our results differ considerably from those of Carlson et al.; we attribute this to the difference in resolution between the two experiments. On the other hand, our results are in quite good agreement with the measurements of Hergenhahn et al. [5] for these features, which were measured at comparable resolution. To our
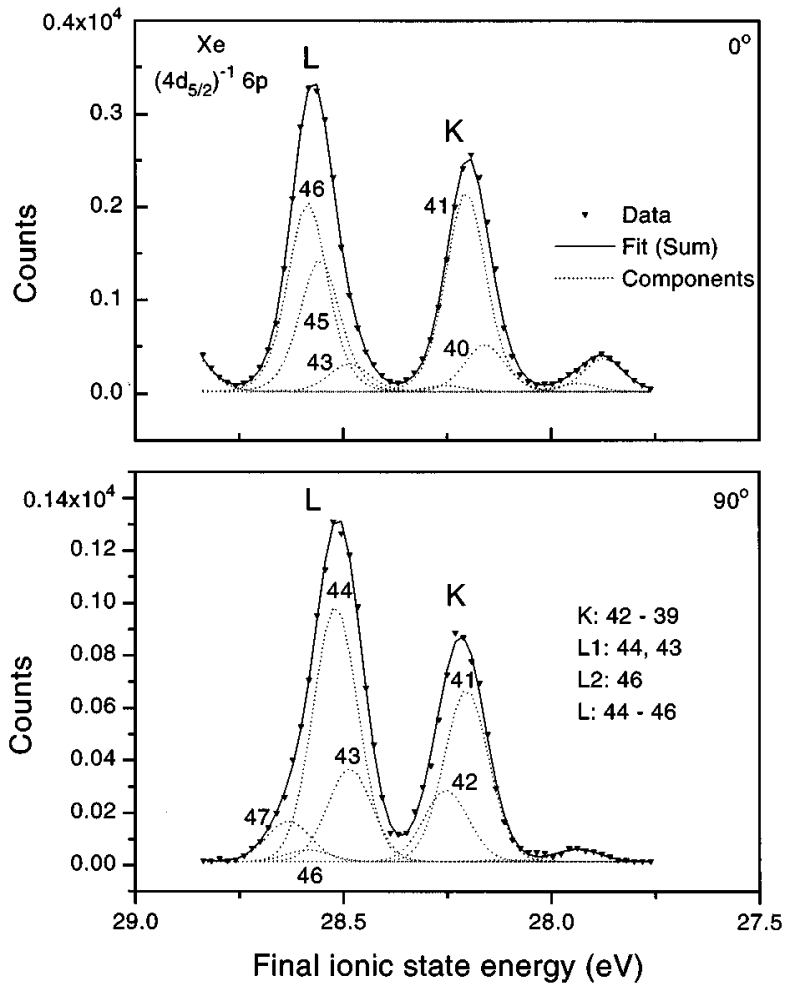

FIG. 3. Relative contributions of the components making up the principal features $K$ and $L$. Peak $L$ may be broken down into $L 1$, which has a large negative $\beta$, and $L 2$, which has a large positive $\beta$. 
knowledge the only other set of data which exist for the $4 d_{3 / 2} \rightarrow 6 p$ excitation is the work of Carlson et al. [4]. Because of the lower resolution in that work, it is difficult to determine exactly which of our lines correspond to their designations. However, based on a comparison of line assignments between Aksela et al. and Carlson et al., we have selected what we believe should be either the same features or superpositions thereof. Results are displayed in Table II. A comparison between the $4 d_{5 / 2}^{-1}$ and $4 d_{3 / 2}^{-1}$ groups in the two tables indicates that the $\beta$ values are similar for most of the equivalent lines, designated by the same letters. However, some $\beta$ values are quite different, especially for the lines $E, F$, and $I$. It is suspected that this is due to a parityunfavored transition contained in one of the $4 d_{5 / 2}^{-1}$ or $4 d_{3 / 2}^{-1}$ transitions that make up the observed features. These are also the lines that have the most widely varying relative intensities in the cross section [1].

The theoretical results $[6,7]$, given in the final two columns of Table I, differ considerably from the experimental results and from each other. We do not understand the source of this discrepancy, although it might arise from a difference in identification of the spin-orbit components. In almost all instances the spin-orbit identifications from the Aksela calculations [1] differ from the assignments of Chen [7]. Because of the very small splittings of many of the spin-orbit features compared to the resolution attainable with the best of modern monochromators, it is likely that it may not be possible to isolate every individual spin-orbit component contributing to these decays. Thus, any measurement will always involve an average over a number of components. The comparison with theoretical results thus requires that the theoretical calculation determine the identity and partial and differential cross sections of each decay feature with extremely good accuracy.

This work was supported by the National Science Foundation under Grant No. PHY-9207634. We acknowledge the experimental efforts of S.B. Whitfield, M.G. Flemming, and A. Fahlman, who helped conduct these measurements as part of a larger project.
[1] H. Aksela, O. P. Sairanen, S. Aksela, A. Kivimäki, A. Naves de Brito, E. Nommiste, J. Tulkki, A. Ausmees, S. J. Osborne, and S. Svensson, Phys. Rev. A 51, 1291 (1995).

[2] M. O. Krause, S. B. Whitfield, C. D. Caldwell, J.-Z. Wu, P. van der Meulen, C. A. de Lange, and R. W. C. Hanson, J. Electron. Spectrosc. Relat. Phenom. 58, 79 (1992)

[3] S. B. Whitfield, C. D. Caldwell, D. X. Huang, and M. O. Krause, J. Phys. B 25, 4755 (1992).

[4] T. A. Carlson, D. R. Mullins, C. D. Beall, B. W. Yates, J. W.
Taylor, D. W. Lindle, and F. A. Grimm, Phys. Rev. A 39, 1170 (1989).

[5] U. Hergenhahn, N. M. Kabachnik, and B. Lohmann, J. Phys. B 24, 4759 (1991).

[6] U. Hergenhahn, B. Lohmann, N. M. Kabachnik, and U. Becker, J. Phys. B 26, L117 (1993).

[7] M. H. Chen, Phys. Rev. A 47, 3733 (1993).

[8] B. Kämmerling, B. Krässig, and V. Schmidt, J. Phys. B 23, 4487 (1990). 